\title{
Comprehensive Esthetic Dentistry
}

Authors: Florin Lazarescu (editor)

Publisher: Quintessence Publishing Co Inc

Language: English

ISSN: 978-1-85097-278-5

Edition: $1 / \mathrm{e}$

Publish Year: 2015

Pages: 364, illustrated

Price: $\$ 228.00$

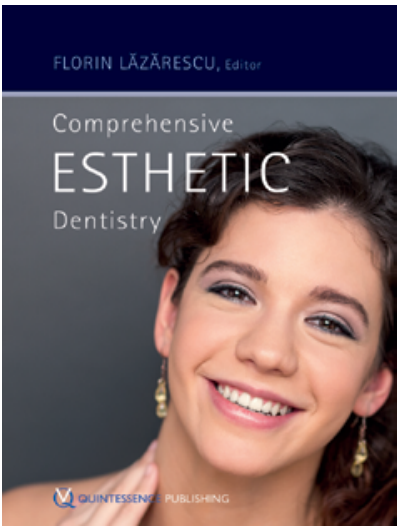

A contemporary dentist has the task of providing his patients with aesthetic treatments, but also in harmony with the other functions of the stomatognathic system. The merit of this book lies with the editor, Dr. Florin Lăzărescu, who managed to raise a group of dental aesthetics specialists who successfully completed this book called "Comprehensive esthetic dentistry".

The book is divided into 15 chapters. The first eight chapters present introductory notions and general principles of dental and dento-facial esthetics, data of photographic examination, communication with the patient in the esthetic assessment integration of the provisional aesthetic rehabilitation in the treatment plan, materials used for aesthetic restorations, notions of minimally invasive procedures in esthetic dentistry, principles of adhesion to dental structures and tooth discoloration. The next seven chapters tackle practical aspects, esthetic restorations of the anterior and lateral teeth, the protocol for adhesive fixations in esthetic restorations, CAD-CAM systems and techniques in the dental office and the interdisciplinary prosthetic- orthodontic-periodontal approach of complex cases requiring teamwork. The book is exceptionally well designed and it gives the practitioner a wealth of useful dental esthetic solutions for both young and experienced doctors. Due to its exceptional qualities to clarify dental esthetics, this book has brought an intense sense of professional achievement and personal satisfaction to the authors and can be seen as a guide in which the practitioner finds an exclusive answer to the problems of dental esthetics that he has to solve in his daily activity.

Marian-Vladimir Constantinescu

DDS, PhD

Holistic Dental \& Medical Institute of Bucharest-ROPOSTURO, Bucharest

Romania e-mail: 